Table 1. Selected Bond Distances ( $(\AA)$ and Angles for $\mathbf{1}$

\begin{tabular}{|c|c|c|c|}
\hline $\mathrm{V}(1)-\mathrm{O}(30)$ & $1.596(8)$ & $\mathrm{V}(8)-\mathrm{O}(13)$ & $1.590(7)$ \\
\hline $\mathrm{V}(1)-\mathrm{O}(12)$ & $1.939(8)$ & $\mathrm{V}(8)-\mathrm{O}(23)$ & $1.957(7)$ \\
\hline $\mathrm{V}(1)-\mathrm{O}(26)$ & $1.945(8)$ & $\mathrm{V}(8)-\mathrm{O}(7)$ & $1.957(8)$ \\
\hline $\mathrm{V}(1)-\mathrm{O}(9)$ & $1.966(7)$ & $\mathrm{V}(8)-\mathrm{O}(1)$ & $1.965(7)$ \\
\hline $\mathrm{V}(1)-\mathrm{O}(19)$ & $1.973(7)$ & $\mathrm{V}(8)-\mathrm{O}(6)$ & 1.971(8) \\
\hline $\mathrm{V}(2)-\mathrm{O}(33)$ & $1.590(8)$ & $\mathrm{V}(9)-\mathrm{O}(36)$ & $1.589(8)$ \\
\hline $\mathrm{V}(2)-\mathrm{O}(2)$ & $1.787(8)$ & $\mathrm{V}(9)-\mathrm{O}(27)$ & $1.772(8)$ \\
\hline $\mathrm{V}(2)-\mathrm{O}(11)$ & $1.840(7)$ & $\mathrm{V}(9)-\mathrm{O}(9)$ & $1.855(7)$ \\
\hline $\mathrm{V}(2)-\mathrm{O}(3)$ & $1.911(7)$ & $\mathrm{V}(9)-\mathrm{O}(7)$ & $1.890(7)$ \\
\hline $\mathrm{V}(2)-\mathrm{O}(17)$ & $1.972(8)$ & $\mathrm{V}(9)-\mathrm{O}(1)$ & $2.002(8)$ \\
\hline $\mathrm{V}(3)-\mathrm{O}(35)$ & $1.598(7)$ & $\mathrm{V}(10)-\mathrm{O}(16)$ & $1.609(7)$ \\
\hline $\mathrm{V}(3)-\mathrm{O}(20)$ & $1.770(8)$ & $\mathrm{V}(10)-\mathrm{O}(4)$ & $1.794(7)$ \\
\hline $\mathrm{V}(3)-\mathrm{O}(1)$ & $1.841(7)$ & $\mathrm{V}(10)-\mathrm{O}(17)$ & $1.827(7)$ \\
\hline $\mathrm{V}(3)-\mathrm{O}(12)$ & $1.924(8)$ & $\mathrm{V}(10)-\mathrm{O}(10)$ & $1.887(8)$ \\
\hline $\mathrm{V}(3)-\mathrm{O}(9)$ & $1.962(8)$ & $\mathrm{V}(10)-\mathrm{O}(11)$ & $1.979(8)$ \\
\hline $\mathrm{V}(4)-\mathrm{O}(32)$ & $1.590(7)$ & $\mathrm{V}(11)-\mathrm{O}(22)$ & $1.591(8)$ \\
\hline $\mathrm{V}(4)-\mathrm{O}(8)$ & $1.724(7)$ & $\mathrm{V}(11)-\mathrm{O}(8)$ & $1.869(7)$ \\
\hline $\mathrm{V}(4)-\mathrm{O}(19)$ & $1.922(8)$ & $\mathrm{V}(11)-\mathrm{O}(21)$ & $1.888(8)$ \\
\hline $\mathrm{V}(4)-\mathrm{O}(2)$ & $1.945(8)$ & $\mathrm{V}(11)-\mathrm{O}(10)$ & $1.993(8)$ \\
\hline $\mathrm{V}(4)-\mathrm{O}(26)$ & $1.971(8)$ & $\mathrm{V}(11)-\mathrm{O}(11)$ & $2.008(8)$ \\
\hline $\mathrm{V}(5)-\mathrm{O}(15)$ & $1.586(9)$ & $\mathrm{V}(12)-\mathrm{O}(34)$ & $1.610(9)$ \\
\hline $\mathrm{V}(5)-\mathrm{O}(14)$ & $1.860(8)$ & $\mathrm{V}(12)-\mathrm{O}(21)$ & $1.717(8)$ \\
\hline $\mathrm{V}(5)-\mathrm{O}(25)$ & $1.874(7)$ & $\mathrm{V}(12)-\mathrm{O}(7)$ & $1.927(8)$ \\
\hline $\mathrm{V}(5)-\mathrm{O}(3)$ & $1.985(7)$ & $\mathrm{V}(12)-\mathrm{O}(27)$ & $1.941(7)$ \\
\hline $\mathrm{V}(5)-\mathrm{O}(17)$ & $2.003(7)$ & $\mathrm{V}(12)-\mathrm{O}(18)$ & $1.982(8)$ \\
\hline $\mathrm{V}(6)-\mathrm{O}(31)$ & $1.614(7)$ & $\mathrm{V}(13)-\mathrm{O}(24)$ & $1.582(7)$ \\
\hline $\mathrm{V}(6)-\mathrm{O}(18)$ & $1.685(8)$ & $\mathrm{V}(13)-\mathrm{O}(14)$ & $1.735(8)$ \\
\hline $\mathrm{V}(6)-\mathrm{O}(23)$ & $1.843(7)$ & $\mathrm{V}(13)-\mathrm{O}(4)$ & $1.939(7)$ \\
\hline $\mathrm{V}(6)-\mathrm{O}(10)$ & $1.887(7)$ & $\mathrm{V}(13)-\mathrm{O}(6)$ & $1.950(7)$ \\
\hline $\mathrm{V}(6)-\mathrm{O}(4)$ & $2.198(9)$ & $\mathrm{V}(13)-\mathrm{O}(23)$ & $1.957(9)$ \\
\hline $\mathrm{V}(7)-\mathrm{O}(28)$ & $1.600(7)$ & $\mathrm{V}(14)-\mathrm{O}(29)$ & $1.595(7)$ \\
\hline $\mathrm{V}(7)-\mathrm{O}(5)$ & $1.715(7)$ & $\mathrm{V}(14)-\mathrm{O}(25)$ & $1.747(8)$ \\
\hline $\mathrm{V}(7)-\mathrm{O}(26)$ & $1.853(8)$ & $\mathrm{V}(14)-\mathrm{O}(12)$ & $1.936(8)$ \\
\hline $\mathrm{V}(7)-\mathrm{O}(3)$ & $1.879(8)$ & $\mathrm{V}(14)-\mathrm{O}(20)$ & $1.940(7)$ \\
\hline $\mathrm{V}(7)-\mathrm{O}(2)$ & $2.142(8)$ & $\mathrm{V}(14)-\mathrm{O}(5)$ & $1.949(7)$ \\
\hline $\mathrm{O}(30)-\mathrm{V}(1)-\mathrm{O}(12)$ & $106.8(4)$ & $\mathrm{O}(13)-\mathrm{V}(8)-\mathrm{O}(23)$ & 107.1(4) \\
\hline $\mathrm{O}(30)-\mathrm{V}(1)-\mathrm{O}(26)$ & $107.1(4)$ & $\mathrm{O}(13)-\mathrm{V}(8)-\mathrm{O}(7)$ & $106.5(4)$ \\
\hline $\mathrm{O}(12)-\mathrm{V}(1)-\mathrm{O}(26)$ & $94.5(3)$ & $\mathrm{O}(23)-\mathrm{V}(8)-\mathrm{O}(7)$ & $94.3(3)$ \\
\hline $\mathrm{O}(30)-\mathrm{V}(1)-\mathrm{O}(9)$ & $107.9(4)$ & $\mathrm{O}(13)-\mathrm{V}(8)-\mathrm{O}(1)$ & $107.3(4)$ \\
\hline $\mathrm{O}(12)-\mathrm{V}(1)-\mathrm{O}(9)$ & $77.3(3)$ & $\mathrm{O}(23)-\mathrm{V}(8)-\mathrm{O}(1)$ & $145.5(3)$ \\
\hline $\mathrm{O}(26)-\mathrm{V}(1)-\mathrm{O}(9)$ & $145.0(3)$ & $\mathrm{O}(7)-\mathrm{V}(8)-\mathrm{O}(1)$ & $78.3(3)$ \\
\hline $\mathrm{O}(30)-\mathrm{V}(1)-\mathrm{O}(19)$ & $108.1(4)$ & $\mathrm{O}(13)-\mathrm{V}(8)-\mathrm{O}(6)$ & 107.1(4) \\
\hline $\mathrm{O}(12)-\mathrm{V}(1)-\mathrm{O}(19)$ & $145.1(3)$ & $\mathrm{O}(23)-\mathrm{V}(8)-\mathrm{O}(6)$ & $76.7(3)$ \\
\hline
\end{tabular}




\begin{tabular}{|c|c|c|c|}
\hline $\mathrm{O}(26)-\mathrm{V}(1)-\mathrm{O}(19)$ & $75.9(3)$ & $\mathrm{O}(7)-\mathrm{V}(8)-\mathrm{O}(6)$ & $146.3(3)$ \\
\hline $\mathrm{O}(9)-\mathrm{V}(1)-\mathrm{O}(19)$ & $91.4(3)$ & $\mathrm{O}(1)-\mathrm{V}(8)-\mathrm{O}(6)$ & $90.9(3)$ \\
\hline $\mathrm{O}(33)-\mathrm{V}(2)-\mathrm{O}(2)$ & $106.1(4)$ & $\mathrm{O}(36)-\mathrm{V}(9)-\mathrm{O}(27)$ & $106.5(5)$ \\
\hline $\mathrm{O}(33)-\mathrm{V}(2)-\mathrm{O}(11)$ & $106.4(4)$ & $\mathrm{O}(36)-\mathrm{V}(9)-\mathrm{O}(9)$ & $108.3(4)$ \\
\hline $\mathrm{O}(2)-\mathrm{V}(2)-\mathrm{O}(11)$ & $99.8(4)$ & $\mathrm{O}(27)-\mathrm{V}(9)-\mathrm{O}(9)$ & $97.9(3)$ \\
\hline $\mathrm{O}(33)-\mathrm{V}(2)-\mathrm{O}(3)$ & $107.1(4)$ & $\mathrm{O}(36)-\mathrm{V}(9)-\mathrm{O}(7)$ & $107.3(4)$ \\
\hline $\mathrm{O}(2)-\mathrm{V}(2)-\mathrm{O}(3)$ & $84.0(3)$ & $\mathrm{O}(27)-\mathrm{V}(9)-\mathrm{O}(7)$ & $84.2(3)$ \\
\hline $\mathrm{O}(11)-\mathrm{V}(2)-\mathrm{O}(3)$ & $143.7(3)$ & $\mathrm{O}(9)-\mathrm{V}(9)-\mathrm{O}(7)$ & $142.0(3)$ \\
\hline $\mathrm{O}(33)-\mathrm{V}(2)-\mathrm{O}(17)$ & $105.2(4)$ & $\mathrm{O}(36)-\mathrm{V}(9)-\mathrm{O}(1)$ & $105.6(4)$ \\
\hline $\mathrm{O}(2)-\mathrm{V}(2)-\mathrm{O}(17)$ & $147.4(3)$ & $\mathrm{O}(27)-\mathrm{V}(9)-\mathrm{O}(1)$ & $146.9(3)$ \\
\hline $\mathrm{O}(11)-\mathrm{V}(2)-\mathrm{O}(17)$ & $79.7(3)$ & $\mathrm{O}(9)-\mathrm{V}(9)-\mathrm{O}(1)$ & $79.2(3)$ \\
\hline $\mathrm{O}(3)-\mathrm{V}(2)-\mathrm{O}(17)$ & 78.4(3) & $\mathrm{O}(7)-\mathrm{V}(9)-\mathrm{O}(1)$ & $79.0(3)$ \\
\hline $\mathrm{O}(35)-\mathrm{V}(3)-\mathrm{O}(20)$ & $107.0(4)$ & $\mathrm{O}(16)-\mathrm{V}(10)-\mathrm{O}(4)$ & $106.5(4)$ \\
\hline $\mathrm{O}(35)-\mathrm{V}(3)-\mathrm{O}(1)$ & $106.6(4)$ & $\mathrm{O}(16)-\mathrm{V}(10)-\mathrm{O}(17)$ & $107.2(4)$ \\
\hline $\mathrm{O}(20)-\mathrm{V}(3)-\mathrm{O}(1)$ & $99.2(3)$ & $\mathrm{O}(4)-\mathrm{V}(10)-\mathrm{O}(17)$ & $98.4(4)$ \\
\hline $\mathrm{O}(35)-\mathrm{V}(3)-\mathrm{O}(12)$ & $108.5(4)$ & $\mathrm{O}(16)-\mathrm{V}(10)-\mathrm{O}(10)$ & $106.8(4)$ \\
\hline $\mathrm{O}(20)-\mathrm{V}(3)-\mathrm{O}(12)$ & $82.9(3)$ & $\mathrm{O}(4)-\mathrm{V}(10)-\mathrm{O}(10)$ & $85.4(4)$ \\
\hline $\mathrm{O}(1)-\mathrm{V}(3)-\mathrm{O}(12)$ & $142.5(3)$ & $\mathrm{O}(17)-\mathrm{V}(10)-\mathrm{O}(10)$ & $143.1(3)$ \\
\hline $\mathrm{O}(35)-\mathrm{V}(3)-\mathrm{O}(9)$ & $105.4(4)$ & $\mathrm{O}(16)-\mathrm{V}(10)-\mathrm{O}(11)$ & $104.1(4)$ \\
\hline $\mathrm{O}(20)-\mathrm{V}(3)-\mathrm{O}(9)$ & $146.1(3)$ & $\mathrm{O}(4)-\mathrm{V}(10)-\mathrm{O}(11)$ & $148.4(3)$ \\
\hline $\mathrm{O}(1)-\mathrm{V}(3)-\mathrm{O}(9)$ & $80.6(3)$ & $\mathrm{O}(17)-\mathrm{V}(10)-\mathrm{O}(11)$ & $79.8(3)$ \\
\hline $\mathrm{O}(12)-\mathrm{V}(3)-\mathrm{O}(9)$ & $77.7(3)$ & $\mathrm{O}(10)-\mathrm{V}(10)-\mathrm{O}(11)$ & $78.3(3)$ \\
\hline $\mathrm{O}(32)-\mathrm{V}(4)-\mathrm{O}(8)$ & $106.0(4)$ & $\mathrm{O}(22)-\mathrm{V}(11)-\mathrm{O}(8)$ & $105.1(4)$ \\
\hline $\mathrm{O}(32)-\mathrm{V}(4)-\mathrm{O}(19)$ & $105.0(4)$ & $\mathrm{O}(22)-\mathrm{V}(11)-\mathrm{O}(21)$ & $103.7(4)$ \\
\hline $\mathrm{O}(8)-\mathrm{V}(4)-\mathrm{O}(19)$ & $95.7(3)$ & $\mathrm{O}(8)-\mathrm{V}(11)-\mathrm{O}(21)$ & $89.7(3)$ \\
\hline $\mathrm{O}(32)-\mathrm{V}(4)-\mathrm{O}(2)$ & $105.8(4)$ & $\mathrm{O}(22)-\mathrm{V}(11)-\mathrm{O}(10)$ & $105.6(4)$ \\
\hline $\mathrm{O}(8)-\mathrm{V}(4)-\mathrm{O}(2)$ & $92.9(3)$ & $\mathrm{O}(8)-\mathrm{V}(11)-\mathrm{O}(10)$ & $148.2(3)$ \\
\hline $\mathrm{O}(19)-\mathrm{V}(4)-\mathrm{O}(2)$ & $144.2(3)$ & $\mathrm{O}(21)-\mathrm{V}(11)-\mathrm{O}(10)$ & $90.9(3)$ \\
\hline $\mathrm{O}(32)-\mathrm{V}(4)-\mathrm{O}(26)$ & $105.6(4)$ & $\mathrm{O}(22)-\mathrm{V}(11)-\mathrm{O}(11)$ & $104.4(4)$ \\
\hline $\mathrm{O}(8)-\mathrm{V}(4)-\mathrm{O}(26)$ & $148.4(3)$ & $\mathrm{O}(8)-\mathrm{V}(11)-\mathrm{O}(11)$ & $89.4(3)$ \\
\hline $\mathrm{O}(19)-\mathrm{V}(4)-\mathrm{O}(26)$ & $76.5(3)$ & $\mathrm{O}(21)-\mathrm{V}(11)-\mathrm{O}(11)$ & 151.1(3) \\
\hline $\mathrm{O}(2)-\mathrm{V}(4)-\mathrm{O}(26)$ & $78.1(3)$ & $\mathrm{O}(10)-\mathrm{V}(11)-\mathrm{O}(11)$ & $75.2(3)$ \\
\hline $\mathrm{O}(15)-\mathrm{V}(5)-\mathrm{O}(14)$ & $103.7(4)$ & $\mathrm{O}(34)-\mathrm{V}(12)-\mathrm{O}(21)$ & $108.8(5)$ \\
\hline $\mathrm{O}(15)-\mathrm{V}(5)-\mathrm{O}(25)$ & $103.8(4)$ & $\mathrm{O}(34)-\mathrm{V}(12)-\mathrm{O}(7)$ & $110.1(4)$ \\
\hline $\mathrm{O}(14)-\mathrm{V}(5)-\mathrm{O}(25)$ & $91.2(4)$ & $\mathrm{O}(21)-\mathrm{V}(12)-\mathrm{O}(7)$ & $141.1(4)$ \\
\hline $\mathrm{O}(15)-\mathrm{V}(5)-\mathrm{O}(3)$ & $105.8(4)$ & $\mathrm{O}(34)-\mathrm{V}(12)-\mathrm{O}(27)$ & $100.0(4)$ \\
\hline $\mathrm{O}(14)-\mathrm{V}(5)-\mathrm{O}(3)$ & $149.4(3)$ & $\mathrm{O}(21)-\mathrm{V}(12)-\mathrm{O}(27)$ & $94.4(3)$ \\
\hline $\mathrm{O}(25)-\mathrm{V}(5)-\mathrm{O}(3)$ & $89.6(3)$ & $\mathrm{O}(7)-\mathrm{V}(12)-\mathrm{O}(27)$ & $78.9(3)$ \\
\hline $\mathrm{O}(15)-\mathrm{V}(5)-\mathrm{O}(17)$ & $104.1(4)$ & $\mathrm{O}(34)-\mathrm{V}(12)-\mathrm{O}(18)$ & $101.2(4)$ \\
\hline $\mathrm{O}(14)-\mathrm{V}(5)-\mathrm{O}(17)$ & $89.1(3)$ & $\mathrm{O}(21)-\mathrm{V}(12)-\mathrm{O}(18)$ & $88.4(4)$ \\
\hline $\mathrm{O}(25)-\mathrm{V}(5)-\mathrm{O}(17)$ & $151.2(4)$ & $\mathrm{O}(7)-\mathrm{V}(12)-\mathrm{O}(18)$ & $84.2(3)$ \\
\hline $\mathrm{O}(3)-\mathrm{V}(5)-\mathrm{O}(17)$ & $76.0(3)$ & $\mathrm{O}(27)-\mathrm{V}(12)-\mathrm{O}(18)$ & $156.4(3)$ \\
\hline $\mathrm{O}(31)-\mathrm{V}(6)-\mathrm{O}(18)$ & $104.2(4)$ & $\mathrm{O}(24)-\mathrm{V}(13)-\mathrm{O}(14)$ & $105.5(4)$ \\
\hline $\mathrm{O}(31)-\mathrm{V}(6)-\mathrm{O}(23)$ & $111.6(4)$ & $\mathrm{O}(24)-\mathrm{V}(13)-\mathrm{O}(4)$ & $106.3(4)$ \\
\hline
\end{tabular}




\begin{tabular}{|c|c|c|c|}
\hline $\mathrm{O}(18)-\mathrm{V}(6)-\mathrm{O}(23)$ & $98.9(4)$ & $\mathrm{O}(14)-\mathrm{V}(13)-\mathrm{O}(4)$ & $91.3(4)$ \\
\hline $\mathrm{O}(31)-\mathrm{V}(6)-\mathrm{O}(10)$ & $110.0(4)$ & $\mathrm{O}(24)-\mathrm{V}(13)-\mathrm{O}(6)$ & $103.4(4)$ \\
\hline $\mathrm{O}(18)-\mathrm{V}(6)-\mathrm{O}(10)$ & $96.3(4)$ & $\mathrm{O}(14)-\mathrm{V}(13)-\mathrm{O}(6)$ & $96.8(4)$ \\
\hline $\mathrm{O}(23)-\mathrm{V}(6)-\mathrm{O}(10)$ & $130.2(3)$ & $\mathrm{O}(4)-\mathrm{V}(13)-\mathrm{O}(6)$ & $145.9(3)$ \\
\hline $\mathrm{O}(31)-\mathrm{V}(6)-\mathrm{O}(4)$ & $96.1(4)$ & $\mathrm{O}(24)-\mathrm{V}(13)-\mathrm{O}(23)$ & $106.6(4)$ \\
\hline $\mathrm{O}(18)-\mathrm{V}(6)-\mathrm{O}(4)$ & $159.6(3)$ & $\mathrm{O}(14)-\mathrm{V}(13)-\mathrm{O}(23)$ & $147.9(3)$ \\
\hline $\mathrm{O}(23)-\mathrm{V}(6)-\mathrm{O}(4)$ & $74.6(3)$ & $\mathrm{O}(4)-\mathrm{V}(13)-\mathrm{O}(23)$ & $78.5(3)$ \\
\hline $\mathrm{O}(10)-\mathrm{V}(6)-\mathrm{O}(4)$ & $74.9(3)$ & $\mathrm{O}(6)-\mathrm{V}(13)-\mathrm{O}(23)$ & $77.2(3)$ \\
\hline $\mathrm{O}(28)-\mathrm{V}(7)-\mathrm{O}(5)$ & $104.2(4)$ & $\mathrm{O}(29)-\mathrm{V}(14)-\mathrm{O}(25)$ & $108.9(4)$ \\
\hline $\mathrm{O}(28)-\mathrm{V}(7)-\mathrm{O}(26)$ & $111.2(4)$ & $\mathrm{O}(29)-\mathrm{V}(14)-\mathrm{O}(12)$ & $111.0(4)$ \\
\hline $\mathrm{O}(5)-\mathrm{V}(7)-\mathrm{O}(26)$ & $96.5(3)$ & $\mathrm{O}(25)-\mathrm{V}(14)-\mathrm{O}(12)$ & $140.1(3)$ \\
\hline $\mathrm{O}(28)-\mathrm{V}(7)-\mathrm{O}(3)$ & $111.1(4)$ & $\mathrm{O}(29)-\mathrm{V}(14)-\mathrm{O}(20)$ & $101.3(4)$ \\
\hline $\mathrm{O}(5)-\mathrm{V}(7)-\mathrm{O}(3)$ & $96.0(3)$ & $\mathrm{O}(25)-\mathrm{V}(14)-\mathrm{O}(20)$ & $94.5(4)$ \\
\hline $\mathrm{O}(26)-\mathrm{V}(7)-\mathrm{O}(3)$ & $131.0(3)$ & $\mathrm{O}(12)-\mathrm{V}(14)-\mathrm{O}(20)$ & $78.3(3)$ \\
\hline $\mathrm{O}(28)-\mathrm{V}(7)-\mathrm{O}(2)$ & $96.8(4)$ & $\mathrm{O}(29)-\mathrm{V}(14)-\mathrm{O}(5)$ & $99.6(4)$ \\
\hline $\mathrm{O}(5)-\mathrm{V}(7)-\mathrm{O}(2)$ & 159.1(3) & $\mathrm{O}(25)-\mathrm{V}(14)-\mathrm{O}(5)$ & $89.7(3)$ \\
\hline $\mathrm{O}(26)-\mathrm{V}(7)-\mathrm{O}(2)$ & $75.9(3)$ & $\mathrm{O}(12)-\mathrm{V}(14)-\mathrm{O}(5)$ & $83.4(3)$ \\
\hline $\mathrm{O}(3)-\mathrm{V}(7)-\mathrm{O}(2)$ & $75.7(3)$ & $\mathrm{O}(20)-\mathrm{V}(14)-\mathrm{O}(5)$ & $156.0(3)$ \\
\hline $\mathrm{V}(3)-\mathrm{O}(1)-\mathrm{V}(8)$ & 139.1(4) & $\mathrm{V}(6)-\mathrm{O}(10)-\mathrm{V}(11)$ & $134.8(4)$ \\
\hline $\mathrm{V}(3)-\mathrm{O}(1)-\mathrm{V}(9)$ & $99.6(3)$ & $\mathrm{V}(2)-\mathrm{O}(11)-\mathrm{V}(10)$ & 99.9(4) \\
\hline $\mathrm{V}(8)-\mathrm{O}(1)-\mathrm{V}(9)$ & $99.0(3)$ & $\mathrm{V}(2)-\mathrm{O}(11)-\mathrm{V}(11)$ & $136.8(4)$ \\
\hline $\mathrm{V}(2)-\mathrm{O}(2)-\mathrm{V}(4)$ & $134.9(4)$ & $\mathrm{V}(10)-\mathrm{O}(11)-\mathrm{V}(11)$ & $100.9(3)$ \\
\hline $\mathrm{V}(2)-\mathrm{O}(2)-\mathrm{V}(7)$ & $97.4(3)$ & $\mathrm{V}(3)-\mathrm{O}(12)-\mathrm{V}(14)$ & $96.8(3)$ \\
\hline $\mathrm{V}(4)-\mathrm{O}(2)-\mathrm{V}(7)$ & $98.1(3)$ & $\mathrm{V}(3)-\mathrm{O}(12)-\mathrm{V}(1)$ & $103.6(4)$ \\
\hline $\mathrm{V}(7)-\mathrm{O}(3)-\mathrm{V}(2)$ & $102.8(4)$ & $\mathrm{V}(14)-\mathrm{O}(12)-\mathrm{V}(1)$ & $142.2(4)$ \\
\hline $\mathrm{V}(7)-\mathrm{O}(3)-\mathrm{V}(5)$ & $136.9(4)$ & $\mathrm{V}(13)-\mathrm{O}(14)-\mathrm{V}(5)$ & $144.8(5)$ \\
\hline $\mathrm{V}(2)-\mathrm{O}(3)-\mathrm{V}(5)$ & $103.9(3)$ & $\mathrm{V}(10)-\mathrm{O}(17)-\mathrm{V}(2)$ & $100.6(4)$ \\
\hline $\mathrm{V}(10)-\mathrm{O}(4)-\mathrm{V}(13)$ & $135.7(4)$ & $\mathrm{V}(10)-\mathrm{O}(17)-\mathrm{V}(5)$ & $137.9(4)$ \\
\hline $\mathrm{V}(10)-\mathrm{O}(4)-\mathrm{V}(6)$ & $95.7(4)$ & $\mathrm{V}(2)-\mathrm{O}(17)-\mathrm{V}(5)$ & $101.0(3)$ \\
\hline $\mathrm{V}(13)-\mathrm{O}(4)-\mathrm{V}(6)$ & $97.2(3)$ & $\mathrm{V}(6)-\mathrm{O}(18)-\mathrm{V}(12)$ & $127.5(4)$ \\
\hline $\mathrm{V}(7)-\mathrm{O}(5)-\mathrm{V}(14)$ & $128.0(4)$ & $\mathrm{V}(4)-\mathrm{O}(19)-\mathrm{V}(1)$ & $103.8(4)$ \\
\hline $\mathrm{V}(13)-\mathrm{O}(6)-\mathrm{V}(8)$ & $102.5(3)$ & $\mathrm{V}(3)-\mathrm{O}(20)-\mathrm{V}(14)$ & $102.0(3)$ \\
\hline $\mathrm{V}(9)-\mathrm{O}(7)-\mathrm{V}(12)$ & $96.7(4)$ & $\mathrm{V}(12)-\mathrm{O}(21)-\mathrm{V}(11)$ & $142.5(5)$ \\
\hline $\mathrm{V}(9)-\mathrm{O}(7)-\mathrm{V}(8)$ & $103.3(4)$ & $\mathrm{V}(6)-\mathrm{O}(23)-\mathrm{V}(13)$ & $109.7(4)$ \\
\hline $\mathrm{V}(12)-\mathrm{O}(7)-\mathrm{V}(8)$ & $142.0(4)$ & $\mathrm{V}(6)-\mathrm{O}(23)-\mathrm{V}(8)$ & $134.2(5)$ \\
\hline $\mathrm{V}(4)-\mathrm{O}(8)-\mathrm{V}(11)$ & $145.8(4)$ & $\mathrm{V}(13)-\mathrm{O}(23)-\mathrm{V}(8)$ & $102.8(3)$ \\
\hline $\mathrm{V}(9)-\mathrm{O}(9)-\mathrm{V}(3)$ & $100.5(3)$ & $\mathrm{V}(14)-\mathrm{O}(25)-\mathrm{V}(5)$ & $142.6(5)$ \\
\hline $\mathrm{V}(9)-\mathrm{O}(9)-\mathrm{V}(1)$ & $138.8(4)$ & $\mathrm{V}(7)-\mathrm{O}(26)-\mathrm{V}(1)$ & $134.4(4)$ \\
\hline $\mathrm{V}(3)-\mathrm{O}(9)-\mathrm{V}(1)$ & $101.2(3)$ & $\mathrm{V}(7)-\mathrm{O}(26)-\mathrm{V}(4)$ & $107.8(4)$ \\
\hline $\mathrm{V}(10)-\mathrm{O}(10)-\mathrm{V}(6)$ & $103.9(4)$ & $\mathrm{V}(1)-\mathrm{O}(26)-\mathrm{V}(4)$ & $103.1(4)$ \\
\hline $\mathrm{V}(10)-\mathrm{O}(10)-\mathrm{V}(11)$ & $104.7(3)$ & $\mathrm{V}(9)-\mathrm{O}(27)-\mathrm{V}(12)$ & $100.3(4)$ \\
\hline
\end{tabular}



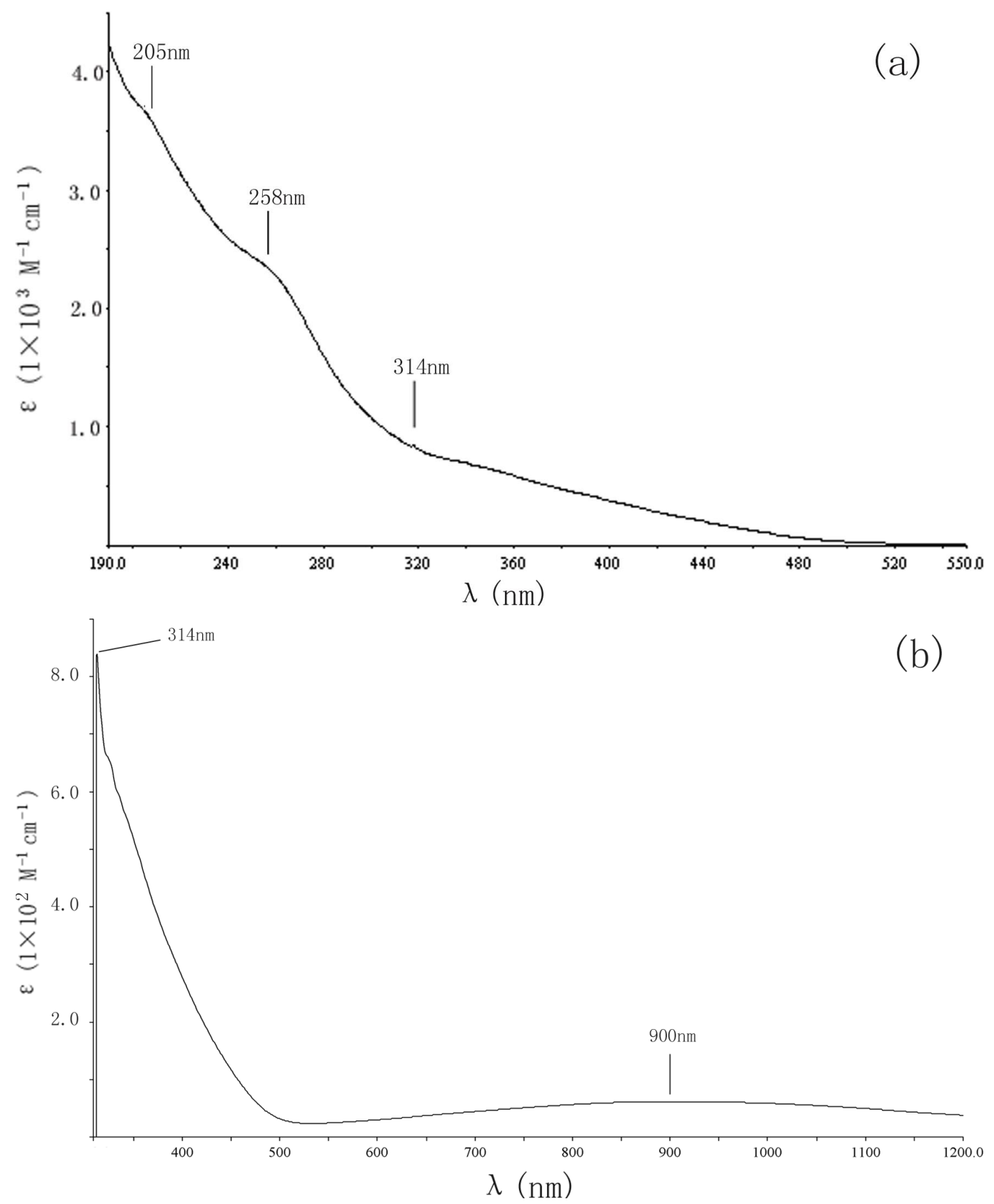

Figure S1 UV/vis absorption spectrum of 1 in $\mathrm{H}_{2} \mathrm{O}$ solution: (a) $0.4 \mathrm{mM}$; (b) $10 \mathrm{mM}$.

\section{Synthesis of $\left[\mathrm{Et}_{4} \mathrm{~N}\right]_{5}\left[\mathrm{~V}_{14} \mathrm{O}_{36} \mathrm{Cl}\right](1)$ and discussions}

Triethylamine $\left(\mathrm{Et}_{3} \mathrm{~N}, 0.11 \mathrm{ml}, 0.8 \mathrm{mmol}\right)$ was slowly added to the mixture of $\mathrm{VO}_{2}(\mathrm{acac})(273 \mathrm{mg}, 1.5 \mathrm{mmol})$ and tetraethylamine chloride $\left(\mathrm{Et}_{4} \mathrm{NCl}, 99 \mathrm{mg}, 0.6\right.$ mmol) in acetonitrile $(50 \mathrm{ml})$ with stirring at room temperature, resulting in a red brown solution. After $6 \mathrm{~h}$, the solvent in reaction solution was removed out under reduced pressure, and then the residue was dissolved in $\mathrm{N}^{\prime} \mathrm{N}$-dimethylformamide (DMF) for crystallization. After filtration, the filtrate was allowed to evaporate slowly 
for a few days, and the black crystals of compound $\mathbf{1}$ suitable for X-ray diffraction were obtained. The crystalline products were collected, washed with diethyl ether and dried in a vacuum to afford $161 \mathrm{mg}$. The yield was $76 \%$ (based on V). Anal. Calc. for $\left[\mathrm{Et}_{4} \mathrm{~N}\right]_{5}\left[\mathrm{~V}_{14} \mathrm{O}_{36} \mathrm{Cl}\right](\mathbf{1}): \mathrm{V} 36.09 ; \mathrm{Cl} 1.79 ; \mathrm{C} 24.32 ; \mathrm{H}$ 5.10; N 3.54\%. Found: V 35.77;

$\mathrm{Cl} 2.02 ; \mathrm{C}, 24.16 ; \mathrm{H}, 5.39 ; \mathrm{N}, 3.37 \%$. IR( $\mathrm{KBr}$, main absorption bands): $2924\left(v_{\mathrm{as}}(\mathrm{C}-\mathrm{H})\right), \quad 2853\left(v_{\mathrm{s}}(\mathrm{C}-\mathrm{H})\right), \quad 1482\left(\delta_{\mathrm{as}}(\mathrm{C}-\mathrm{H})\right), \quad 1456\left(\delta_{\mathrm{as}}(\mathrm{C}-\mathrm{H})\right), \quad 1396\left(\delta_{\mathrm{s}}(\mathrm{C}-\mathrm{H})\right)$, $1384\left(\delta_{\mathrm{s}}(\mathrm{C}-\mathrm{H})\right), 1099(v(\mathrm{C}-\mathrm{N})), 1031(v(\mathrm{C}-\mathrm{C})), \quad 979(v(\mathrm{~V}=\mathrm{O})), 838(v(\mathrm{O}-\mathrm{V}-\mathrm{O}))$, and $605(\mathrm{v}(\mathrm{O}-\mathrm{V}-\mathrm{O})) \mathrm{cm}^{-1}$

In this synthesis, we employed $\mathrm{VO}_{2}(\mathrm{acac})$ as a soluble $\mathrm{VO}_{2}{ }^{+}$source and the amine as a Lewis base in order to adjust the reaction conditions. It appears that the use of $\mathrm{VO}_{2}(\mathrm{acac})$ is crucial to the synthesis of compound $\mathbf{1}$, for we found that other soluble vanadium(V) precursors, such as $\mathrm{VO}(\mathrm{OEt})_{3}$ and $\mathrm{VO}(\mathrm{OMe})_{3}$, lead to mix-valence polyoxovanadates that are structurally different from compound 1. The electrical conductivity measurement for compound $\mathbf{1}$ in the solid state was performed, but no appreciable conductivity was observed.

\section{References:}

(5) (a) Müller, A.; Sessoli, R.; Krickemeyer, E.; Bögge, H.; Meyer, J.; Gatteschi, D.; Pardi, L.; Westphal, J.; Hovemeier, K.; Rohlfing, R.; Doring, J.; Hellweg, F.; Beugholt, C.; Schmidtmann, M. Inorg. Chem. 1997, 36, 5239-5250. 